\title{
Sex ratio distortion in the Nesolynx thymus (Hymenoptera: Eulophidae), an ecto-pupal parasitoid of uzifly, Exorista sorbillans (Diptera: Tachinidae)
}

\author{
Bandekodigenahalli M. PRAKASH ${ }^{1}$, Aswathaiah PRATHimA², Hoolageri C. HUCHESH², Hemagirigowda \\ RAVIKUMAR ${ }^{2}$, SHANKARANARAYAN SAMPATHKUMAR² and HoSAGAVI P. PUTTARAJU ${ }^{2}$
}

\author{
${ }^{1}$ Evolutionary Biology Laboratory, Evolutionary and Organismal Biology Unit, Jawaharlal Nehru Centre for Advanced Scientific \\ Research, Jakkur PO, Bangalore 560064, Karnataka, India; e-mail: prakashbm@gmail.com \\ ${ }^{2}$ Department of Biological Sciences, Bangalore University, Bangalore 560056, Karnataka, India; e-mails: prathima2805@gmail.com; \\ hucheshbt@gmail.com; ravikumarh79@gmail.com; sam82kumar@gmail.com; puttarajuhp@hotmail.com
}

Key words. Hymenoptera, Eulophidae, Diptera, Tachinidae, Wolbachia, Nesolynx thymus, Exorista sorbillans, sex ratio distortion, parthenogenesis, temperature treatment

\begin{abstract}
The reproductive alterations induced by maternally inherited $\alpha$-proteo-bacteria Wolbachia to their hosts is a well-documented phenomenon. In Nesolynx thymus, a gregarious hymenopterous ecto-pupal parasitoid of the uzifly, Exorista sorbillans, diagnostic PCR assay using specific primers revealed the presence of Wolbachia. Following genetic crossing experiments, we observed a female biased sex ratio of $1: 9.5$ at $25^{\circ} \mathrm{C}$ and $1: 3$ male to female ratio when the populations were exposed to heat shock $33^{\circ} \mathrm{C}$ for six hours. Furthermore, we found infection polymorphism, where female parasitoids are infected by Wolbachia but males are not infected. Infected eggs develop into females, whereas uninfected eggs develop parthenogenetically into males. The results are discussed in the context of the possible mechanism of sex-ratio bias caused by Wolbachia.
\end{abstract}

\section{INTRODUCTION}

Wolbachia is a genus of maternally inherited intracellular $\alpha$-proteobacteria which infect a wide range of arthropods and nematodes (Werren, 1997; Braig et al., 1998; Stouthamer et al., 1999), estimated to occur in about $66 \%$ of all known insect species (Hilgenboecker et al., 2008). They are abundant intracellular symbionts and have attracted significant attention in terms of their ability to manipulate host reproduction through cytoplasmic incompatibility (CI), feminization, induction of parthenogenesis and male killing (Werren, 1997; Stouthamer et al., 1999). In CI, infected males are incompatible with uninfected females or females infected with some other strains of Wolbachia; however, infected females are compatible with infected or uninfected males (Yen \& Bar, 1971; O’Neill \& Karr, 1990). This is the most commonly observed Wolbachiainduced reproductive phenotype in most of insect orders. Feminization is the reproductive phenotype whereby all genetic males are converted into functional females, and is found in some terrestrial isopods as well as one particular species of butterfly (Rigaud et al., 1991; Kageyama et al., 2002). However, in other lepidopteran species such as the corn borer, Ostrinia scapulalis (Crambidae), Wolbachia causes lethal feminization of genotypic males (Kageyama \& Traut, 2004; Sugimoto \& Ishikawa, 2012). During parthenogenesis, unfertilized eggs develop into males (arrehenotoky) or females (thelytoky) (Werren et al., 2008). Each of these reproductive anomalies enhances female production and hence the reproduction of the bacterium and is collectively referred to as "reproductive parasitism" (Werren et al., 2008).
The effect of Wolbachia depends on a number of factors, including host genetic background, atmospheric temperature, resource quality and host age. These factors directly affect Wolbachia densities within its host, which in turn have context specific effects on the respective host populations. Among these, temperature has a remarkable effect upon Wolbachia, associated bacteriophage, involved in horizontal gene transfer (Bordenstein \& Wernegreen, 2004) and on host population dynamics (Bordenstein \& Werren, 2003; Bordenstein \& Bordenstein, 2011; Kraaijeveld et al., 2011).

Nesolynx thymus (Hymenoptera: Eulophidae) is an ectopupal-gregarious parasitoid of the "uzifly", Exorista sorbillans (Diptera: Tachinidae), itself an endo-larval parasitoid of the silkworm moth, Bombyx mori L. (Lepidoptera: Bombycidae), and accounting for some $8-10 \%$ yield loss in India and other silk growing countries. $N$. thymus is often used as a primary biocontrol agent to regulate the populations in nature (Narayanaswamy \& Devaiah, 1998). Earlier investigations have associated the efficacy of this agent with its phenomenal host searching ability and parasitization capacity (Kumar et al., 1993; Narayanaswamy \& Devaiah, 1998). The successful expansion of the parasitoid is based on its ability to tolerate highly variable temperatures ranging from 15 to $35^{\circ} \mathrm{C}$ (Jyothi et al., 1993). The overall developmental time of the parasitoid from egg to adult is around 16 days, whilst gravid females can produce around 300 offspring during their lifetime. Interestingly, the sex ratio of the parasitoid has been found to vary considerably between studies (ChannaBasavanna et al., 1993; Aruna \& Manjunath, 2010). In addition to its use as a biocontrol agent against uzifly, $N$. thymus also hyper-parasitizes 
Bleparipha zebena (Walker) (Diptera: Tachinidae), a larval endo-parasitoid of the tasar silkworm, Antheraea mylitta (Drury) (Saturniidae) and a primary parasitoid of the house fly, Musca domestica L. (Muscidae) the blow fly Chrysomya rufifacies (Calliphoridae) as well as an unidentified calliphorid fly (Channabasavanna et al., 1993; Narayanaswamy \& Devaiah, 1998). During mass rearing, the house fly is used as an inexpensive and easy to rear host (Aruna \& Manjunatha, 2010).

Over the last 25 years, considerable efforts have been made to curtail the uzifly through chemical, physical and biological methods (Narayanaswamy \& Devaiah, 1998). Many parasitoids and microbial agents have been studied (Narayanaswamy \& Devaiah, 1998; Puttaraju \& Prakash, 2005a, b) and currently, more than 20 species belonging to the families Chalcididae, Diapriidae, Encrytidae, Eulophidae and Pteromalidae have been identified as ecto/endo, larval/pupal or solitary/gregarious parasitoids of this fly. These hymenopterans inflict some $82 \%$ of the biocontrol mortality with the remaining achieved through predatory insects and vertebrate predators and some by pathogenic bacteria (Narayanaswamy \& Devaiah, 1998; Puttaraju \& Prakash, 2005a, b). Because of the prominence of $N$. thymus and the need to improve production, the reproductive behavior of the $N$. thymus and possible interactions with the thelytoky inducing Wolbachia endosymbiont were presently examined. Furthermore, since there is no experimental evidence that Wolbachia has any effect on sex ratio in the parasitoid, only circumstantial correlation in terms of sex ratio bias, this aspect was also investigated. To this end, we for the first time screened Wolbachia infection in $N$. thymus using a diagnostic PCR approach in order to determine whether or not the bacteria do play a role in the reproduction of the parasitoid.

\section{MATERIAL AND METHODS}

\section{Collection and rearing of parasitoid $N$. thymus}

Around 1,000 parasitoids were collected from the Ramanagara silkworm cocoon market. These were maintained in the laboratory on uzifly pupae at a ratio of approximately 1 parasitoid : 10 hosts. Ten discrete generations were maintained in the laboratory to eliminate any variation in the population due to environmental variations in three 1.01 conical flasks, covered with muslin cloth, with $\sim 250$ parasitoids per flask in the first generation followed by $\sim 500-800$ parasitoids per flask in subsequent generations. Each flask contained a separate individual population indicated as blocks 1, 2 and 3. Two days after emergence from the host pupae, females in the flask were provided with 250 -uzifly pupae per flask for one day and monitored until emergence in separate conical flasks. Populations were maintained in an insect rearing chamber at a temperature of $25 \pm 1^{\circ} \mathrm{C}$ and $80-85 \% \mathrm{RH}$ and 12L : 12D conditions.

\section{Genomic DNA isolation and PCR amplification}

Individual male and female parasitoid DNA was extracted using ZR Insect/Tissue DNA kit-5TM (Zymo Research, Irvine, CA, USA). Whole insects were ground in ZR Bashing Bead ${ }^{\mathrm{TM}}$ lysis tube and homogenized in $600 \mu \mathrm{l}$ lysis solution, DNA being extracted according to the manufacturer's protocol, with at least 15 males and females used per population. The dried DNA pellet was re-suspended in $25 \mu 110 \mathrm{mM}$ TE buffer, pH 8.0. DNA was quantified using a spectrophotometer (Bio-Rad SmartSpec ${ }^{\mathrm{TM}}$ plus) and stored at $-20^{\circ} \mathrm{C}$ for further use.

The polymerase chain reaction (PCR) assay was used to amplify a Wolbachia surface protein (WSP) gene using the primer pair: wsp $81 F$ 5'-TGG TCC AAT AAG TGA TGA AGA AAC3'and wsp691R 5'-AAA AAT TAA ACG CTA CTC CA-3', which amplify around $630 \mathrm{bp}$ (Braig et al., 1998). This was done in an Eppendrof thermocycler in $20 \mu \mathrm{l}$ reaction volume containing $1 \times$ PCR buffer, $0.2 \mathrm{mM}$ dNTP's, $2.5 \mathrm{mM} \mathrm{MgCl}_{2}$, and 0.5 unit Taq DNA polymerase (MBI-Fermentas, Amherst, NY, USA), $0.1 \mu \mathrm{M}$ of each forward and reverse primer, $20 \mathrm{ng}$ of template DNA and a final volume of sterile water to make up a total of $20 \mu 1$ reaction mixture. PCR conditions were: initial denaturation at $94^{\circ} \mathrm{C}$ for 3 min followed by 40 cycles with denaturing at $94^{\circ} \mathrm{C}$ for $1 \mathrm{~min}$, primer annealing at $55^{\circ} \mathrm{C}$ for $1 \mathrm{~min}$, primer extension at $72^{\circ} \mathrm{C}$ for $2 \mathrm{~min}$, followed by a final extension step at $72^{\circ} \mathrm{C}$ for $10 \mathrm{~min}$. For positive and negative controls, uzifly and silkworm DNA was used, respectively (Prakash \& Puttaraju, 2007). In addition, an insect specific $18 \mathrm{~S}$ rDNA primer pair amplifying a $555 \mathrm{bp}$ segment of the host genome (18S F1 5'-TTG GAG GGCAAG TCT GGT GC-3' and 18S R1 5'-ACT TCG GCG GAT CGC TAG CT-3') were used to determine the quality of the DNA extraction (Wenseleers \& Billen, 2000). PCR products were separated on a $1.2 \%$ agarose gel run in $1 \times$ TBE buffer, $\mathrm{pH} 8.0$ for a length of 5-6 $\mathrm{cm}$ at constant 65 volts. The gel was stained with $0.5 \mu \mathrm{g} / \mathrm{ml} \mathrm{gel}$ ethidium bromide just prior to casting. A $1 \mathrm{~kb}$ standard molecular weight marker (Chromous Biotech ${ }^{\mathrm{TM}}$, Bangalore, India) was used to estimate amplified band size.

\section{Crossing experiments}

Parasitoids were sexed following emergence. Single female and male parasitoids were placed in $100 \mathrm{ml}$ conical flasks covered with muslin cloth and provided with 20 two day old uzifly pupae. The host pupae were replaced every day for five days and parasitized pupae maintained in separate $500 \mathrm{ml}$ conical flasks for each block until the emergence of $N$. thymus, adult parasitoids being sexed each day after emergence. For each block, 20 replicate crosses were maintained, whilst each flask was provided with a $50 \%$ honey solution on cotton balls as food for the adult parasitoids (Aruna \& Manjunath, 2010).

\section{Heat shock treatment}

About 250 one day old parasitoids from each block were exposed to $33^{\circ} \mathrm{C}$ heat shock treatment for six hours in an incubator. Following heat shock, parasitoids were maintained at $25^{\circ} \mathrm{C}$ for one more day and crosses then made as shown above with 20 replications from each block.

\section{Data analysis}

Experimental data were pooled and block means, standard deviations and errors calculated. Means were compared by chisquare testing. Since chi-square tests provided only 4 degree of freedom, a correction value of 0.5 was subtracted from the deviation value before squaring (Khan \& Khanum, 1994). To test the null hypothesis, the calculated values of chi-square were compared with the table value at $5 \%$ and $1 \%$ confidence levels.

\section{RESULTS AND DISCUSSION}

In all three populations, female $N$. thymus proved PCR positive for Wolbachia and yielded the expected band size of around $630 \mathrm{bp}$, whereas males were negative (Fig. 1). In addition, insect specific $18 \mathrm{~S}$ rDNA primer yielded an expected band of around $550 \mathrm{bp}$ which confirmed the quality of insect DNA used for amplification of Wolbachia. The genetic crossing results revealed that the sex ratio was bi- 


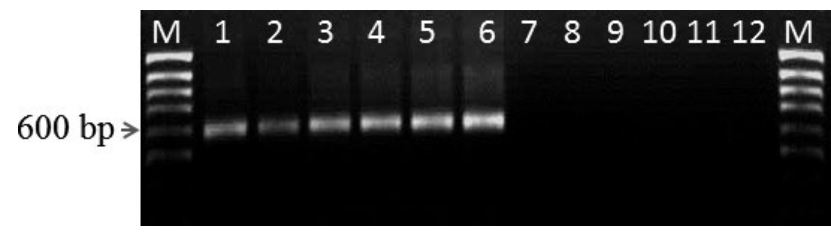

Fig. 1. Gel photograph showing amplification of the Wolbachia wsp gene sequence in Nesolynx thymus. Lane M: $1 \mathrm{~kb}$ molecular weight marker; lanes 1,2 and 3: Wolbachia in heat shocked parasitoid and lanes 4, 5 and 6 normal female $N$. thymus of blocks 1 , 2 and 3, respectively; Lanes 7, 8, 9, and 10, 11, 12: males of heat shocked and normal $N$. thymus in blocks 1, 2 and 3, respectively.

ased towards females, with the number of offspring varying from 83 to 406, and with an average of 217.7. The number of males and females were $20.6 \pm 3.5$ and $197.1 \pm 11.9$, respectively, i.e., a ratio of $1: 9.5$ (Fig. 2) at $25^{\circ} \mathrm{C}$, significantly different from a $1: 1$ ratio at $P=0.01$ using a chisquare test $\left(x^{2}=116.93 ; P<0.001\right)$.

In addition, temperature treatment for $6 \mathrm{~h}$ at $33^{\circ} \mathrm{C}$ on one-day-old adult parasitoids revealed a reduction in the total offspring number of $23.5 \%$ (they produced an average of 166.6 offspring/ female) compared to parasitoids reared at $25^{\circ} \mathrm{C}$. The sex ratio of offspring of temperature treated parasitoids was on average 40.7 males and 125.9 females (approximately a ratio of 1 male to 3 females) and significantly different from a $1: 1$ ratio at $P=0.01\left(x^{2}=44.542\right.$; $P<0.01)$. However, the female bias was reduced in heat shock treatment, compared to parasitoids reared at $25^{\circ} \mathrm{C}$ (Fig. 2). PCR assay confirmed the absence of Wolbachia in male offspring, whose parents had been exposed to heat shock, and thus produced a greater number of males, in turn indicating that temperature had a negative effect on the parasitoid-Wolbachia association. It would be interesting to rear parasitoids at different temperatures for one or more complete generations to quantify the effects of this treatment on Wolbachia and its host. Our hypothesis is that the infected eggs of $N$. thymus may develop into females, while uninfected eggs develop into males. But we cannot ruled out the possibility of low Wolbachia infection in males which may not be detected through standard PCR approaches.

Earlier Aruna \& Manjunath (2010) reported a 1:17 male to female ratio in $N$. thymus; however, they had not considered the possible presence of Wolbachia and its effects. This could simply be due to arrhenotoky, as is typical of Hymenoptera. However, the sex ratio varies between studies which in turn might be due to variation in the level of Wolbachia infection, a possibility these authors seemingly had not considered. High Wolbachia infection rates in females, which facilitates infection of more eggs, leads to the production of more females. In the case of lower Wolbachia levels, asynchronous transmission may result in fewer uninfected eggs, which induces parthenogenetic male production.

Wolbachia is also sometimes known to increase host fitness and fecundity (Dedeine et al., 2001; Puttaraju \& Prakash, 2005a, b, 2009). This characteristic is being exploited in several biocontrol programs (e.g. Brelsfoard \&

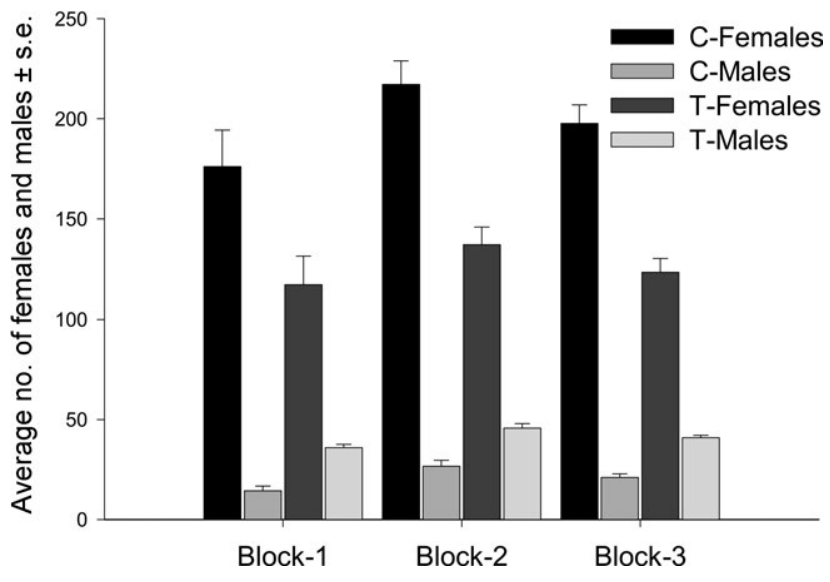

Fig. 2. Sex ratio in the Nesolynx thymus an ecto-pupal-parasitoid of uzifly, Exorista sorbillans. Average numbers of females and males in normal $(\mathrm{C})$ and temperature treated $(\mathrm{T})$ crosses are on the Y-axis and the blocks are on the X-axis. Twenty replications were performed per each block/populations for normal and heat shock treatment populations. Error bars represents standard errors (s.e.) of the means.

Dobson, 2009); therefore, probably Wolbachia could be used for improvement of reproductive performance in $N$. thymus. Our screening studies suggest that the bacterium infecting $N$. thymus is associated with female biased sex ratio. Although it may prove difficult, other novel fecundity and life span enhancing strains of Wolbachia could be introduced depending upon the cytoplasmic background from other established host species, achievable through embryonic microinjection (Sampath Kumar \& Puttaraju, 2012). Future studies on the phylogenetic relationship of Wolbachia in $N$. thymus and its dipterous host uzifly, E. sorbillans, may provide an interesting insight into these evolutionary aspects. The E. sorbillans- $N$. thymus-Wolbachia host-parasitoid-symbiont association may well provide an ideal platform for such evolutionary studies, because, E. sorbillans and N. thymus both harbour Wolbachia, whilst at the same time exhibiting different pattern of reproductive anomalies (i.e. biased sex ratio). Uzifly exhibit Wolbachia-induce cytoplasmic incompatibility, sex ratio distortion and fecundity reduction, but in N. thymus, female biased sex ratios have been observed. $N$. thymus continually parasitizes E. sorbillans (Narayanaswamy \& Devaiah, 1998) and therefore, there may well be horizontal transmission occurring between host and parasitoid. If such transmission indeed occurs, what the reproductive effects induced by Wolbachia on uzifly and its parasitoid might be are questions that need to be answered.

In conclusion, the present study is the first report of Wolbachia infection in Nesolynx thymus, infection that is most probably responsible for the female biased sex-ratios typically seen in this species, whereas as presently found, males are uninfected by the bacterium.

ACKNOWLEDGEMENTS. The authors thank K. Kraaijeveld and one anonymous reviewer for their various constructive comments, which have greatly helped improve the MS and H.D. Loxdale for his helpful editorial suggestions. BMP thanks the Department of Science and Technology of the Government of 
India for financial assistance via a Fast-Track Young Scientist fellowship.

\section{REFERENCES}

Aruna A.S. \& Manjunath D. 2010: Reproductive performance of $N$. thymus (Hymenoptera: Eulophidae) as influenced by host (Musca domestica) size. - BioControl 55: 245-252.

Bordenstein S.R. \& Bordenstein S.R. 2011: Temperature affects the tripartite interaction between bacteriophage WO, Wolbachia, and cytoplasmic incompatibility. - PLOS ONE 6: e29106. doi:10.1372/journal.pone.0029106.

Bordenstein S.R. \& Wernegreen J.J. 2004: Bacteriophage flux in endosymbionts (Wolbachia): Infection frequency, lateral transfer, and recombination rates. - Mol. Biol. Evol. 21: 1981-1991.

Bordenstein S.R. \& WerRen J.H. 2003: Host genotype determines cytoplasmic incompatibility type in the haplodiploid genus Nasonia. - Genetics 146: 223-233.

Braig H.R., Zhou W.G., Dobson S.L. \& O’Neill S.L. 1998: Cloning and characterization of a gene encoding the major surface protein of the bacterial endosymbiont Wolbachia pipientis. —J. Bacteriol. 180: 2373-2378.

Brelsfoard C.L. \& Dobson S.L. 2009. Wolbachia-based strategies to control insect pests and disease vectors. - Asia Pac. J. Mol. Biol. Biotechnol. 17: 55-63.

ChannaBasavanna G.P., Veeranna G. \& Dandin S.B. (eds) 1993: Recent Advances in Uzifly Research: Proceedings of National Seminar on Uzifly and its Control, 16-17 January, 1992. Jwalamukhi Job Press, Bangalore, 305 pp.

Dedeine F., Vavre F., Fleury F., Loppin B., Hochber M.E. \& Boultreau M. 2001: Removing symbiotic Wolbachia bacteria specifically inhibits oogenesis in a parasitic wasp. - Proc. Natl. Acad. Sci. USA 98: 6247-6252.

Hilgenboecker K., Hammerstein P., Schlattmann P., Telschow A. \& Werren J.H. 2008: How many species are infected with Wolbachia? A statistical analysis of current data. - FEMS Microbiol. Lett. 281: 215-220.

Jyothi H.K., Veeranna G., Nirmala M.R. \& Bali G. 1993: Relative efficacy of the parasitoids of uzifly as biocontrol agent. In ChannaBasavanna G.P., Veeranna G. \& Dandin S.B. (eds): Recent Advances in Uzifly Research: Proceedings of National Seminar on Uzifly and its Control, 16-17 January, 1992. Jwalamukhi Job Press, Bangalore, pp. 107-115.

Kageyama D. \& Traut W. 2004: Opposite sex-specific effects of Wolbachia and interference with the sex determination of its host Ostrinia scapulalis. - Proc. R. Soc. Lond. (B) 271: 251-258.

Kageyama D., Nishimura G., Hoshizaki S. \& Ishikawa Y. 2002: Feminizing Wolbachia in an insect, Ostrinia furnacalis (Lepidoptera: Crambidae). - Heredity 88: 444-449.

Khan I.A. \& Khanum A. 1994: Fundamentals of Biostatistics. Ukaaz, Hyderabad, pp. 392-431.
Kraaijeveld K., Reumer B.M., Mouton L., Kremer N., Vavre F. \& VAN AlPhen J.J.M. 2011: Does a parthenogenesis-inducing Wolbachia induce vestigial cytoplasmic incompatibility. Nawissenschaften 98: 175-180.

Kumar P., Manjunath D. \& Datta R.K. 1993: Biological control of uzifly. In ChannaBasavanna G.P., Veeranna G. \& Dandin S.B. (eds): Recent Advances in Uzifly Research: Proceedings of National Seminar on Uzifly and its Control, 16-17 January, 1992. Jwalamukhi Job Press, Bangalore, pp. 91-106.

Narayanaswamy K.C. \& Devaiah M.C. 1998: Silkworm Uzifly. Zen, Bangalore, $231 \mathrm{pp}$.

O’NeILL S.L. \& KarR T.L. 1990: Bidirectional incompatibility between conspecific populations of Drosophila simulans. Nature 348: 178-180.

Prakash B.M. \& Puttaraju H.P. 2007: Infection frequency of A and B supergroup Wolbachia in insects and pests associated with mulberry and silkworm. - J. Biosci. 32: 671-676.

Puttaraju H.P. \& Prakash B.M. 2005a: Effects of Wolbachiatargeted tetracycline on host-parasitoid-symbiont interaction. - Eur. J. Entomol. 102: 669-674.

Puttaraju H.P. \& Prakash B.M. 2005b: Wolbachia and reproductive conflicts in the uzi fly, E. sorbillans (Diptera: Tachinidae). - Arch. Insect Biochem. Physiol. 60: 230-235.

Puttaraju H.P. \& Prakash B.M. 2009: Effects of elimination of Wolbachia on the oogenesis of the uzifly, E. sorbillans, a parasitoid of the silkworm, Bombyx mori. - Entomol. Res. 39: 372-379.

Rigaud T., Juchault P. \& Mocquard J.P. 1991: Experimental study of temperature effects on the sex ratio of broods in terrestrial Crustacea Armadiliidium vulgure Latr. Possible implications in natural populations. - J. Evol. Biol. 4: 603-617.

Sampath Kumar S. \& Puttaraju H.P. 2012: Improvised microinjection technique for mosquito vectors. - Indian J. Med. Res. 136: 971-978.

Stouthamer R., Breeuwer J.A.J. \& Hurst G.D.D. 1999: Wolbachia pipientis: Microbial manipulator of arthropod reproduction. - Annu. Rev. Microbiol. 53: 71-102.

Sugimoto T.N. \& IshiKawa Y. 2012: A male-killing Wolbachia carries a feminizing factor, which is associated with degradation of the sex determining system of its host. - Biol Lett. 8: 412-415.

Wenseleers T. \& Billen J. 2000: No evidence for Wolbachiainduced parthenogenesis in the social Hymenoptera. - J. Evol. Biol. 13: 277-280.

WERREN J.H. 1997: Biology of Wolbachia. - Annu. Rev. Entomol. 32: 287-602.

Werren J.H., Baldo L. \& Clark M.E. 2008: Wolbachia: master manipulator of invertebrate biology. - Nat. Rev. Microbiol. 6: 741-751.

YeN J.H. \& BARR A.R. 1971: New hypothesis of the cause of cytoplasmic incompatibility in Culex pipiens. - Nature 232: 657-658.

Received December 25, 2013; revised and accepted April 22, 2014 Prepublished online August 13, 2014 